${ }^{1}$ Faculty of Chemistry and Pharmaceutical Sciences, University of Chile, CHILE

${ }^{2}$ Clinical Hospital, University of Chile, CHILE ${ }^{3}$ Faculty of Pharmacy, University of Valparaiso, CHILE

\title{
Role of Stress and Sympathetic Innervation in the Development of Polycystic Ovary Syndrome
}

\section{Contents}

Abstract

Origin and Ovarian Distribution of Sympathetic Nerves

Nerve Activation During Polycystic Ovary

Effect of Stress and $\beta$-Adrenergic System on the Formation of Ovarian Cyst

Concluding Remarks

References

\section{Abstract}

This work attempts to give a review of ovarian innervation, the mechanism of regulation of nerve activity and the role of the sympathetic activity in ovarian pathologies affecting reproductive function. We provide a succinct outline of the findings of our group in this area. The participation of stress as an etiological factor for ovarian pathologies throughout animal models and preliminary data in patients with polycystic ovary syndrome give strong support for a participation of sympathetic nerves in the ovary function both in normal and pathological states.

Keywords: ovary, stress, polycystic ovary, sympathetic innervation, hiperandrogenemia.

Invited Mini-review

\section{Correspondence}

Hernán E Lara

Department of Biochemistry and Molecular Biology, Faculty of Chemistry and Pharmaceutical Sciences, Universidad de Chile, Santiago, Chile.

E-mail:_hlara@ll.ciq.uchile.cl

Financial Support: Fondecyt 1020581, DID Sal 002/2; ECOS/Conicyt, DIPUV 6/98 and DIPUV 26/2003. 


\section{Origin and Ovarian Distribution of Sympathetic Nerves}

Postganglionic sympathetic fibers innervating the ovary derive from neuronal cell bodies of the ovarian ganglion, which is located at the origin of the ovarian artery, and from cell bodies of the celiac and renal plexuses (Baljet \& Drukker, 1979; reviewed by Burden, 1985). In the rat, the ovary receives its sympathetic innervation from two sources: (a) the ovarian plexus nerve (PN) which travels along the ovarian artery, and (b) the superior ovarian nerve ( $\mathrm{SON}$ ) which is associated with the suspensory ligament (Lawrence \& Burden, 1980). In general, the SON fibers innervate preponderantly the secretory components of the ovary, i.e., interstitial glands and follicles, whereas the PN fibers are mostly perivascular (Lawrence \& Burden, 1980).

The intraovarian distribution of sympathetic fibers is similar in all species, but the density of the network varies considerably among species (Burden, 1985). Importantly, the fibers are associated with the vasculature, travel along the interstitial tissue and surround developing follicles, but penetrate neither the corpus luteum nor the granulosa cell layer of follicles. The ovary's sympathetic innervation rapidly recovers after the organ's transplantation to an ectopic site (Lara et al., 1991). More recently, we found that the rat ovarian penetration of nerve fibers is accompanied by a complete biochemical biosynthetic system as ovarian norepinephrine (NE) content returns to control values 28 days after surgical denervation of the SON (Lara et al., 2002). This capacity of the ovary is probably due to the trophic support exerted by abundant amounts of nerve growth factor (NGF) and its receptors present within the gland. Immunoblockade of NGF actions during the early postnatal period not only deprives the ovary from sympathetic nerves, but also renders it incapable of reinnervation during adulthood (Lara et al., 1991).

Morphological studies, in which ovarian sympathetic nerves were visualized by histofluorescence methods, suggested that the fibers were mainly noradrenergic (Jacobowitz \& Wallach, 1967; Owman et al., 1967). This notion was later confirmed by selective biochemical measurement of ovarian catecholamines (Bahr \& Ben-Jonathan, 1981), a study which also showed that epinephrine and dopamine, though detectable, constitute a minor fraction of ovarian catecholamines. However, some differences exist between species; there are high levels of dopamine found in the human ovary (Lara et al., 2001). A possible physiological role of ovarian dopamine needs to be clarified because of the presence of $\mathrm{D}_{1}$-dopaminergic receptors in human granulosa cells, and its coupling to the intracellular third messenger, dopamine and cAMP-related phosphoprotein, $\mathrm{M}_{\mathrm{r}}$ 32,000 (DARPP-32) phosphorylation (Mayerhofer et al., 1999; Mayerhofer et al., 2000).

\section{Nerve Activation During Polycystic Ovary}

Polycystic ovary (PCO) syndrome is widely recognized as the most common cause of infertility in women. PCO syndrome is a complex disease characterized by anovulatory failure and the presence of ovarian cyst, amenorrhea, hyperandrogenemia 
and variable levels of circulating gonadotropins (Yen, 1999). We have obtained evidences that an hyperactivation of sympathetic nerves, arriving to the ovary, participates in the development and maintenance of polycystic ovary in the rat.

Lara et al. (1993) found that PCO induced by the administration of a single dose of estradiol valerate to rats results in profound changes in ovarian catecholamine homeostasis, which were initiated before the development of cyst and persist after cyst were formed. These changes include increased ovarian noradrenaline (NE) content, enhanced NE release from ovarian nerve terminals and down-regulation of $\beta$-adrenoceptors in theca-interstitial cells, the ovarian compartment directly innervated by sympathetic nerves. The increase in NE released found in PCO rats within 30 days of estradiol administration is even more pronounced after 60 days, i.e., when ovarian cyst were fully developed. The increase in NE-induced release found 30 days after estradiol administration could be the result of an increase in the size of the NE pool to be released and this effect has to involve changes in the synthesis and release of neurotransmitters.

The increased activity of sympathetic nerves during development of PCO in rats is accompanied with a striking enhanced ovarian steroidal responsiveness to both $B$ adrenoceptor and gonadotropins stimulation and this abnormal response can be prevented by selectively ablating the neural input to endocrine cells of the ovary (Barria et al., 1993). It is noteworthy that progesterone and androgens secretory response of cystic ovaries to isoproterenol were enhanced in the face of a reduced $\beta$-adrenoceptor content. A similar paradox was noted when studying the progesterone response to zinterol, a $\beta_{2}$ adrenoceptor agonist during the first proestrus at puberty (Ojeda \& Aguado, 1985) and the progesterone response to isoproterenol during adult estrus rats (Barria et al., 1993). In both cases the $\beta-$ adrenoceptor content was reduced, but the activation of the remaining receptors resulted in a much greater stimulation of progesterone secretion than in other phases of the cycle. Probably coupling of $\beta$-adrenoceptors to adenylyl cyclase is increased during PCO. The constitutive activation of adenylyl cyclase in McCune-Albright syndrome also produces an increased ovarian steroid output and unilateral formation of follicular cysts (Weinstein et al., 1991). The activation of noradrenergic outflow to the ovary observed in animals with PCO suggests that an abnormally heightened sympathetic tone to the gland underlies the steroidal hyperresponsiveness of PCO. The restoration of estrous ciclicity and ovulation resulting from ablation of the SON (Barria et al., 1993), which carries the bulk of the sympathetic innervation to ovarian endocrine cells (Lawrence \& Burden, 1980), further implies a neural abnormality but now in the maintenance of PCO condition.

Although these data gives strong evidence for an involvement of the nervous system in the development and maintenance of PCO they do not provide however, evidence if it was the overdose of estradiol the primary stimulus that initiates the syndrome and nerve activation was only a correlation between multiples changes induced by estradiol in the ovary. The activation of ovarian sympathetic nerves is driven by an increase of the intraovarian expression of the genes encoding nerve growth factor (NGF), and its low- 
affinity receptor, p75 NGF receptor. Immunoblockade of NGF actions and intraovarian administration of an antisense oligodeoxynucleotide to p75NGFR, partially restored estrous cyclicity and ovulatory capacity decreased during PCO induction to rats (Lara et al., 2000). On the other hand, intraovarian grafting of genetically modified cells to overexpress the gene for NGF, increased the incidence of precystic follicles accompanied by a reduced number of healthy antral follicles. Therefore, an abnormally elevated production of NGF suffices to initiate several of the structural and functional alterations associated with the development of ovarian cysts (Dissen et al., 2000).

\section{Effect of Stress and B-Adrenergic System on the Formation of Ovarian Cyst}

To discriminate from a direct effect of estradiol as a primary etiologic factor in cyst formation, Paredes et al. (1998) have tested the capacity of stress to activate sympathetic nerves and to induce changes in follicular development and cyst formation. Because kinetics studies using mouse, hamster and rat have shown that the large preovulatory follicles ovulating in response to LH surge actually enter the growing pool of follicles around 20 days earlier (Greenwald \& Roy, 1994), it was used a chronic intermittent cold and restraint stress during 3 and 11 weeks ( 3 h/day, from Monday to Friday). The combined stress procedure induced an increase in the sympathetic nerve activity of the ovary and also in corticosterone plasma levels and catecholamine content in the adrenal gland. Although the increase in NE found in the celiac ganglion represents a general effect of stress on the sympathetic nerves similar to that described at the superior cervical ganglion after restraint stress (Nankova et al., 1996), the local effect of stress in the ovary was demonstrated by the increase in the release in ${ }^{3} \mathrm{H}-\mathrm{NE}$ recently taken up by the ovary. The increase in the activity of the autonomic nerves was also correlated with the appearance of precystic follicles in the ovary. Longer period of stress did not develop more changes in follicular development. Instead of this, after 11 weeks of stress there was no cyst formation and even a recovery in sympathetic nerve activity to control values was found. The participation of corticosterone from adrenal origin could be the responsible for the reversion of the stress effect on ovarian physiology. In this support, an inhibitory effect of corticosterone on NE synthesis has been demonstrated (Axelrod \& Reisine, 1984). In addition, Galvez et al. (1999) found that adrenalectomy by its own, increased ${ }^{3} \mathrm{H}-\mathrm{NE}$ release and up-regulated $\mathrm{B}$-adrenoceptors of the ovary. A further increase in the release of NE appeared when the same procedure of stress was applied to adrenalectomized rats. These data strongly suggest that chronic stress is able to increase sympathetic nerve activity and that the increase in nerve activity is associated with changes in follicular development and the appearance of precystic structures in the ovary. We have recently studied the effect of a chronic intermittent cold stress procedure on sympathetic nerve activation and its effect to ovarian physiology (Dorfman et al., 2003). This paradigm $\left(4^{\circ} \mathrm{C}\right.$ during $3 \mathrm{~h}$ /day, from Monday to Friday for 3 or 4 weeks) did not affect basal corticosterone 
plasma levels. At 3 weeks of stress, we detected a decrease of NE in the ovary but after 4 weeks of stress the ovarian neurotransmitter increased over the values of control unstressed rats. Follicular development was modified during the stress procedure. A decrease in preantral healthy follicles with no atresia was found at 3 and 4 weeks of stress. In parallel with the increase in NE content in the ovary at 4 week of stress, we observed a recovery in antral follicles and the appearance of a new population of follicles presenting a hypertrophied theca cell layer characteristically seen in patients with PCO syndrome (Dorfman et al., 2003).

Because stress response is a multifactor event that involves complex neuroendocrine responses, we have recently applied a method to directly stimulate $\beta$ adrenoceptors by in vivo administration of the B-adrenoceptor agonist isoproterenol (Lara et al., 2002). We used the administration of isoproterenol $(125 \mu \mathrm{g} / \mathrm{kg} /$ day $)$ during 10 days, a procedure previously described to induce cardiac hypertrophy in the rat (Benjamin et al., 1989), to study the changes induced by $B$-adrenoceptor overstimulation in ovarian follicular development. Because of the time needed to complete the follicular development (Greenwald \& Roy, 1994), we choose three different times to study: immediately after finishing the treatment and 20 or 30 days after finishing the treatment. After isoproterenol administration finished, the ovary had already developed follicular cyst. The increased number of cyst was maintained all 20 days after the end of the treatment, but a clear increase in the number of cyst was found after 30 days of finishing the $B$-adrenoceptor agonist administration. Probably the mechanism involved in this process is associated to a hyperandrogenism condition evoked by the chronic $\beta$-adrenoceptor stimulation induced by isoproterenol, because the ovary of these rats presented an increased capacity to secrete androgens when it was incubated in vitro (Lara et al. 2002). It is interesting to mention that the improved secretory capacity of androgens of the ovary is only presented while the Badrenoceptor agonist is present because the production of androgens by the ovary returned to control levels after 20 and 30 days of the end of the treatment. Thus, a chronic $\beta$ adrenoceptor agonist induced increase in androgens production could induce an aberrant follicular development, that finally conduced the ovary to develop cyst in a process that is fully expressed after 30 days of the end of isoproterenol administration.

Sympathetic nerve activation, either by stress procedures or in vivo administration of isoproterenol, increased the number of ovarian cyst in the rat. Our recent findings (Lara et al., 2001), that biopsies of human ovaries release NE in vitro when the tissue is electrically stimulated and that this secretion of $\mathrm{NE}$ is coupled to steroid production through activation of $\beta_{2}$ - adrenoceptor binding sites present at ovarian secretory cells, strongly suggest that a neural-mediated activation of ovarian steroid secretion and intraovarian neurotrophic influences on follicular development, could also be principal components in the development of the PCO in humans.

More recently, and to analyze the relation between sympathetic nerve activity, chronic stress and the presence of PCO syndrome, we studied the depression and stress 
score in patients with PCO syndrome by internationally accepted techniques to relate changes in the nervous activity with the appearance of PCO syndrome. We studied 18 patients, from the Gynecology and Endocrinology section of the University of Chile Clinical Hospital, diagnosed clinically and biochemically with PCO syndrome. Twelve healthy women with regular menstrual cycles were studied as control. The Goldberg score to measure anxiety, and the proportion of patients with score higher than 11 were higher in PCO syndrome than in control patients $(10.4 \pm 5.1$ vs. $4.8 \pm 5.3$, PCO vs. controls, $\mathrm{p}<0.005)$, (44\% vs. $17 \%$, PCO vs. controls, $\mathrm{p}<0.01)$. Two PCO syndrome patients, but none of the controls, presented depression (Hamilton test). The higher anxiety level was positively correlated with basal insulin and HOMA test $(r=0.52, p=0.02 ; r=0.58, p=0.01)$.

\section{Concluding Remarks}

The variety of evidence presented in this chapter strongly suggests that a chronic increase in ovarian sympathetic nerve activity is related to changes in follicular development, producing non-cyclic anovulatory ovary that develops cysts. The process seems to be reversible if the sympathetic activity is attenuated. This data could offer a new alternative to treat the PCO syndrome using $\beta$-adrenoceptor antagonists in patients resistant to the standard procedure of clomiphene citrate treatment and provides a non expensive means to reinstate ovulatory cycles in PCO syndrome-affected women. On the other hand, the new data in patients with PCO syndrome showing the positive correlation between increase in anxiety and incidence in PCO syndrome warning the importance to manage the stress in patients to decrease the impact in the maintenance of PCO syndrome and its well-recognized metabolic complications associated with stress and PCO.

\section{References}

Axelrod J, Reisine TD. Stress hormones: their interactions and regulation. Science 1984:224;452-8.

Baljet B, Drukker J. Extrinsic innervation of the abdominal organs in the female rat. Acta Anat 1979:104;243-67.

Bahr JM, Ben-Jonathan N. Elevated catecholamines in porcine follicular fluid before ovulation. Endocrinology 1985:117;620-3.

Barria A, Leyton V, Ojeda SR, Lara HE. Ovarian steroidal response to gonadotropins and $\beta$-adrenergic stimulation is enhanced in polycystic ovary syndrome: Role of sympathetic innervation. Endocrinology 1993:133;2696-703.

Benjamin IJ, Jalil JE, Tan LB, Cho K, Weber KT, Clark WA. Isoproterenol-induced myocardial fibrosis in relation to myocyte necrosis. Circulation Res 1989:65;657-70.

Burden HW. The adrenergic innervation of mammalian ovaries. In: Ben-Jonathan N, Bahr JM, Weiner RI (eds). Catecholamines as Hormone Regulators, New York: Serono Simposia Publications, Raven Press; 1985:261-78.

Dissen GA, Lara HE, Leyton V, Paredes A, Hill DF, Costa ME, Martinez-Serrano A, 
Ojeda SR. Intraovarian excess of nerve growth fractor increases androgens secretion and disrupts estrous cyclicity in the rat. Endocrinology 2000:141;1073-82.

Dorfman M, Arancibia S, Fiedler JL, Lara HE. Chronic intermittent cold stress activates ovarian sympathetic nerves and modifies ovarian follicular development in the rat. Biol Reprod 2003:68;2038-43.

Galvez A, Paredes A, Fiedler JL, Venegas M, Lara HE. Effects of adrenalectomy on the stress-induced changes in ovarian sympathetic tone in the rat. Endocrine 1999:10;131-5.

Greenwald GS, Roy SK. Follicular development and its control. In: Knobil E and Neill JD, editors. The physiology of reproduction. New York: Raven Press; 1994:629-724.

Jacobowitz D, Wallach EE. Histochemical and chemical studies of the autonomic innervation of the ovary. Endocrinology 1967:81;1132-9.

Lara HE, Dees WL, Hiney JK, Dissen GA, Rivier C, Ojeda SR. Functional recovery of the developing rat ovary after transplantation: Contribution of the extrinsic innervation. Endocrinology 1991:129;1849-60.

Lara HE, Ferruz JL, Luza S, Bustamante D, Borges Y, Ojeda SR. Activation of ovarian sympathetic nerves in polycystic ovary syndrome. Endocrinology 1993:133;2690-5.

Lara HE, Dissen GA, Leyton V, Paredes A, Fuenzalida H, Fiedler JL, Ojeda SR. An increased intraovarian synthesis of nerve growth factor and its low-affinity receptor is a principal component of steroid-induced polycystic ovary in the rat. Endocrinology 2000:141;1059-72.

Lara HE, Porcile A, Espinoza J, Romero C, Luza SM, Fuhrer J, Miranda C, Roblero J. Release of norepinephrine from human ovary. Coupling to steroidogenic response. Endocrine 2001:15;187-92.

Lara HE, Dorfman M, Venegas M, Luza SM, Luna SL, Mayerhofer A, Guimaraes MA, Rosa E Silva AA, Ramirez VD. Changes in sympathetic nerve activity of the mammalian ovary during a normal estrous cycle and in polycystic ovary syndrome: Studies on norepinephrine release. Microsc Res Tech 2002:59;495-502.

Lawrence IE, Burden HW. The origin of the extrinsic adrenergic innervation to the rat ovary. Anat Rec 1980:196;51-9.

Mayerhofer A, Hemmings HC, Snyder GL, Greengard P, Boddien S, Berg U, Brucker C.. Functional dopamine-1 receptors and DARPP-32 are expressed in human ovary and granulose cells in vitro. J Clin Endocrinol Metab 1999:84;257-64.

Mayerhofer A, Fritz S, Grünert R, Sanders S, Duffy DM, Ojeda SR, Stouffer RL. D1receptor, DARPP-32 and PP-1 in the primate corpus luteum and luteinized granulose cells: Evidence for phosphorylation of DARPP-32 by dopamine and human chorionic gonadotropin. J Clin Endocrinol Metab 2000:85;4750-7.

Nankova B, Kvetnansky, Hiremagalur B, Sabban B, Rusnak M, Sabban EL. Immobilization stress elevates expression for catecholamine biosynthetic enzymes and some neuropeptides in rat sympathetic ganglia: Effects of adrenocorticotropin and glucocorticoides. Endocrinology 1996:137;5597-604. 
Ojeda SR, Aguado LI. Adrenergic control of the prepubertal ovary: Involvement of local innervation and circulating catecholamines. In: Ben-Jonathan N, Bahr JM, Weiner RI (eds). Catecholamines as Hormones Regulators, New York: Serono Symposia Publications, Raven Press; 1985:293-310.

Owman CH, Rosengreen E, Sjoberg NO. Adrenergic innervation of the human female reproductive organs: a histochemical and chemical investigation. Obstet Gynecol 1967:30;763-73.

Paredes A, Gálvez A, Leyton V, Aravena G, Fiedler JL, Bustamante D, Lara HE. Stress promotes development of ovarian cyst in rats. The possible role of sympathetic nerve activation. Endocrine 1998:8;309-15.

Weinstein LS, Shenker A, Gejman PV, Merino MJ, Friedman R, Spiegel AM. Activating mutations of the stimulatory $G$ protein in the McCune-Albright syndrome. N Engl J Med 1991:325;1688-95.

Yen SSC. Polycystic ovary syndrome (hyperandrogenic chronic anovulation). In: Yen SSC, Jaffe RB (eds). Reproductive Endocrinology, Philadelphia, WB Saunders Co; 1999:436-76. 